Bojar, Hans

[25]

\section{In situ monitoring of early effects of epirubicin-based neoadjuvant chemotherapy in breast cancer by CDNA array technology}

\author{
Hans Bojar ${ }^{1}$, Hans Bernd Prisack ${ }^{1}$, Olga Modlich ${ }^{1}$, \\ Mohmoud Danaei ${ }^{2}$, Jörg Rahnenführer ${ }^{3} \&$ Werner Audretsch ${ }^{2}$ \\ ${ }^{1}$ Department of Chemical Oncology, HHU Düsseldorf, Düsseldorf, Germany \\ ${ }^{2}$ Comprehensive Breast Center, Municipal Hospital Düsseldorf, Düsseldorf, \\ Germany \\ ${ }^{3}$ Mathematical Institute, Bioinformatics Group
}

A subset of breast cancer patients ( $14 \%$ of our data set) neoadjuvantly treated with epirubicin-based chemotherapy experience complete remission associated with prolonged survival. Based on massively parallel transcriptome analysis, we describe for the first time early in situ effects of epirubicin-based chemotherapy in a neoadjuvant setting. We took jet-needle biopsies from 22 individual tumors immediately before and $24 \mathrm{~h}$ after the first cycle of chemotherapy. Clontech's 1.2 human cancer complementary DNA arrays were used. Jet-needle biopsies (30 $\mathrm{mm}^{3}$ ) yielded sufficient material (20-60 $\mu \mathrm{g}$ total RNA) for gene expression profiling. Of 1,185 genes selected for the array $30-50 \%$ were expressed. The most prominent early effect of chemotherapy was an upregulation of the cyclin kinase inhibitor WAF1, an important regulator of the G1-S transition in $82 \%$ of the tumors (average induction 2.8-fold; range 1.5- to 7-fold). Of the other cyclin kinase inhibitors, only p16-INK4A and p57-KIP2 were upregulated in a small subset of tumors. We observed a moderate chemotherapy-induced upregulation of various DNA repair enzymes. This group included XPC, an initiator of global genome nucleotide excision repair; endonuclease III homologue 1 (HNTH1) and DNA-damage-inducible transcript 3 (DDIT3, GADD153). Preliminary supervised cluster analysis seems to be encouraging for response prediction. Jetneedle technology is appropriate for in situ response monitoring in neoadjuvantly treated breast cancer patients. Chemotherapy-induced effects on cellcycle control and DNA repair could be demonstrated as early as $24 \mathrm{hrs}$ after the onset of therapy.

Bonnet, Jacques

\section{Doxorubicin-induced alterations of the gene expression profiles of leukemia cells in culture using cDNA microarrays}

Antoine Vekris ${ }^{1}$, François Godard 2,3 , Marie-Christine Haaz ${ }^{3}$, Jacques Robert ${ }^{1,2} \&$ Jacques Bonnet ${ }^{1,2}$

${ }^{1}$ Universite de Bordeaux 2, Bordeaux, France

${ }^{2}$ Institut Bergonié, Bordeaux, France

${ }^{3}$ DiGEM S.A.

We have studied the effects of doxorubicin on the gene expression profiles of the K562 cell line and its doxorubicin-resistant variant, K562DoxR using hybridization of labeled complementary DNAs obtained from the messenger RNA population of treated and untreated cells with 1,176 cDNA probes spotted on Atlas membranes (Clontech). We added doxorubicin to cell cultures in the exponential phase of growth at a concentration corresponding to its $\mathrm{IC}_{50}$ value in each cell line ( $1.2 \mu \mathrm{M}$ for K562 cells, $32 \mu \mathrm{M}$ for K562DoxR cells). Exposure times were 2, $4,8,24$ and $48 \mathrm{~h}$. We first checked that the relative signal intensities were reproducible for the hybridization step (average of $1,176 \mathrm{CVs}=16 \%$ ) as well as for the retrotranscription and hybridization steps taken together (average of 1,176
$\mathrm{CVs}=18 \%$ ). The transcriptome was strongly altered by drug treatment, the alterations generally increasing with drug exposure time. In K562 cells after $24 \mathrm{~h}$ of drug treatment 18 genes out of 1,176 seemed to be at least fivefold overexpressed and 38 at least fivefold underexpressed. After $48 \mathrm{~h}$ these figures were 97 and 228, respectively. Among the most affected genes were those encoding growth factors and their receptors, transcription factors and genes related to DNA repair and apoptosis. These preliminary results allow the identification of the functions involved in cell response to doxorubicin. The kinetic analysis of the gene expression profiles may yield insights into the sequence of the initial events leading to cell death or cell resistance after drug exposure.

Bourdon, Veronique

[27]

\section{A $12 p$ array to identify amplified and overexpressed sequences in testicular germ cell tumors}

Veronique Bourdon ${ }^{1}$, Musa M. Mhlanga ${ }^{2}$, Aldo Massimi ${ }^{3}$ Sanjay Tyagi ${ }^{2}$, Raju Kucherlapati ${ }^{3} \&$ Raju S.K. Chaganti ${ }^{1}$

${ }^{1}$ Human Genetics Department, Memorial Sloan-Kettering Cancer Center, 1275 York Avenue, New York, New York 10021, USA

${ }^{2}$ Department of Molecular Genetics, Public Health Research Institute, 455 First Avenue, New York, New York 10016, USA

${ }^{3}$ Molecular Genetics Department, Albert Einstein College of Medicine, 1300 Morris Park Avenue, Bronx, New York 10461, USA

Testicular germ cell tumors (TGCTs) show, for the most part, a characteristic cytogenetic aberration carrying one or more copies of $i(12 p)$. Mostly i $(12 p)$-negative TGCTs also show an overrepresentation of $12 \mathrm{p} 11 \mathrm{p} 12$ sequences. Critical genes in the amplicon defined by $12 \mathrm{p} 11 \mathrm{p} 12$ may be involved in the progression of TGCTs and other tumors. To identify directly the expressed sequence tags (ESTs) involved in the amplicon, we performed comparative genomic hybridization array analysis of five TGCTs demonstrated to exhibit high DNA copy number in this region. The use of arrays spotted with 8,060 IMAGE complementary DNA clones amplified by means of the polymerase chain reaction (PCR) identified 18 amplified ESTs. Most of them were mapped to $12 \mathrm{p}$ and the remainder have been mapped to 12. We also identified new testis-associated transcripts by PCR with reverse transcription of the amplified EST sequences. Using combined RNA-array and real-time-beacon PCR analysis, we identified two amplified and overexpressed ESTs. The level of expression in these ESTs was four- to sixfold higher in one tumor sample compared with normal testis. The expression ratios obtained by real-time-beacon PCR analysis correlated with those obtained by RNA-array data analysis, thus validating the techniques used. However, we failed to find an amplified and overexpressed EST commonly overexpressed in all the tumor samples tested, perhaps because the arrays used might only represent approximately $10 \%$ of human genes. Therefore we decided to design a $12 p$ array containing 768 ESTs, representing all the 12p-mapped ESTs found in the available databases. Results of comparative genomic hybridization and RNA-array analysis of TGCTs using the $12 \mathrm{p}$ array will be presented. 